Cardiology in the Young (2017), 27, 217-223 C Cambridge University Press, 2016. This is an Open Access article, distributed under the terms of the Creative Commons Attribution licence (http://creativecommons.org/licenses/by/4.0/), which permits unrestricted re-use, distribution, and reproduction in any medium, provided the original work is properly cited.

doi:10.1017/S1047951116001645

\title{
The neurodevelopmental implications of hypoplastic left heart syndrome in the fetus
}

\author{
David F. A. Lloyd, ${ }^{1,2}$ Mary A. Rutherford, ${ }^{2}$ John M. Simpson, ${ }^{1}$ Reza Razavi ${ }^{1,2}$ \\ ${ }^{1}$ Paediatric Cardiology Department, Evelina Children's Hospital; ${ }^{2}$ Division of Imaging Sciences and Biomedical \\ Engineering, King's College London, London, United Kingdom
}

\begin{abstract}
As survival after cardiac surgery continues to improve, an increasing number of patients with hypoplastic left heart syndrome are reaching school age and beyond, with growing recognition of the wide range of neurodevelopmental challenges many survivors face. Improvements in fetal detection rates, coupled with advances in fetal ultrasound and MRI imaging, are contributing to a growing body of evidence that abnormal brain architecture is in fact present before birth in hypoplastic left heart syndrome patients, rather than being solely attributable to postnatal factors. We present an overview of the contemporary data on neurodevelopmental outcomes in hypoplastic left heart syndrome, focussing on imaging techniques that are providing greater insight into the nature of disruptions to the fetal circulation, alterations in cerebral blood flow and substrate delivery, disordered brain development, and an increased potential for neurological injury. These susceptibilities are present before any intervention, and are almost certainly substantial contributors to adverse neurodevelopmental outcomes in later childhood. The task now is to determine which subgroups of patients with hypoplastic left heart syndrome are at particular risk of poor neurodevelopmental outcomes and how that risk might be modified. This will allow for more comprehensive counselling for carers, better-informed decision making before birth, and earlier, more tailored provision of neuroprotective strategies and developmental support in the postnatal period.
\end{abstract}

Keywords: Fetal cardiology; paediatric cardiology; CHD; hypoplastic left heart syndrome; neurodevelopment

Received: 3 November 2015; Accepted: 31 August 2016; First published online: 8 November 2016

S URGICAL OUTCOMES FOR PATIENTS WITH CHD HAVE traditionally been measured in terms of shortterm morbidity and mortality. As survival from cardiac surgery continues to improve, robust data are emerging on the prevalence of a wide range of neurodevelopmental impairments in up to half of survivors, ${ }^{1}$ with univentricular conditions such as hypoplastic left heart syndrome among the highest risk. ${ }^{1-6}$ Indeed, the burden of impairments in these patients has prompted some groups to suggest that neurodevelopmental outcomes should be one of the

Correspondence to: Dr D. F. A. Lloyd, MBChB, MRCPCH, MPhil, Clinical Research Fellow (Paediatric \& Fetal Cardiology), Division of Imaging Sciences and Biomedical Engineering, 4th Floor, Lambeth Wing, St. Thomas Hospital, Westminster Bridge Road, London, SE1 7TH, United Kingdom. Tel: +44 20 7188 7188; Fax: +44 207188 5442; E-mail: david.lloyd@kcl.ac.uk primary outcome measures in clinical decision making for patients with $\mathrm{CHD}^{7}$

The potential causative and contributory factors are myriad. Many $\mathrm{CHDs}$ require corrective or palliative surgery in the neonatal period, and the use of cardiopulmonary bypass, deep hypothermic circulatory arrest, and various surgical and postoperative factors have all been implicated in contributing to adverse neurodevelopmental outcomes. ${ }^{3,8-10}$ The palliative approach used in univentricular conditions necessitates multiple obligatory surgeries, persistently abnormal haemodynamics, and lifelong cardiovascular morbidity. ${ }^{11}$ Chromosomal and other syndromic conditions associated with $\mathrm{CHD}$ are independently associated with impaired neurodevelopmental status. ${ }^{12}$ Other genetic factors, for example, expression of neuroprotective apolipoprotein E, may help modulate brain injury 
following surgery in certain patients. ${ }^{13}$ Socio-economic and parental factors also influence later intellectual development. $^{14,15}$ Increasingly, however, both preoperative and prenatal brain dysgenesis, immaturity, and white matter injury are being recognised in patients with CHD. ${ }^{16,17}$

In the last few decades, major advances have been made in diagnosing structural heart defects before birth, and anatomical variables that are likely to contribute to poor surgical outcomes are more effectively diagnosed than ever before. ${ }^{18}$ The ability to identify fetuses and neonates with pre-intervention brain abnormalities, reduced cerebral blood flow, or other risk factors associated with later neurological impairments may also help identify subgroups of patients at high risk of poor neurodevelopmental outcomes. This could have important consequences for prenatal counselling, which often focusses on fetal risk factors affecting surgical mortality, even though these may in fact have little effect on parental decision making. ${ }^{19}$ It may also help identify infants at increased risk in the early neonatal and perioperative period, in whom novel neuroprotective techniques may be of particular benefit. ${ }^{20}$

This manuscript reviews existing data regarding neurodevelopmental outcomes in patients with hypoplastic left heart syndrome, with particular attention to factors encountered in the fetus or neonate before surgical intervention that may have a bearing on later brain development.

\section{Hypoplastic left heart syndrome and neurodevelopmental outcomes}

Hypoplastic left heart syndrome is a spectrum of congenital cardiac anomalies in which the left-sided heart chambers are small or absent with a hypoplastic ascending aorta and arch. This results in the inability of the left heart to support the systemic circulation in postnatal life.

Norwood et $\mathrm{al}^{21}$ performed the first successful staged surgical palliation in a group of patients operated on from 1979 to 1981; however, in most centres, it was well over a decade before the procedure was performed in significant numbers. Thus, an increasing number of patients with hypoplastic left heart syndrome are now reaching school age and early adulthood, with consequent recognition of the significant neurodevelopmental challenges many survivors face. Over $30 \%$ of infants with hypoplastic left heart syndrome experience moderate to severe neurodevelopmental impairments, ${ }^{4}$ including deficits in gross and fine psychomotor development, hypotonia, behavioural problems, microcephaly, and global developmental delay. ${ }^{22}$ School-aged children often manifest motor deficiencies such as poor balance, coordination, and dexterity; behavioural issues such as shyness or inattention; and significant cognitive challenges. ${ }^{22}$ Indeed, up to a third of these children will receive some form of special education, with around one in five scoring an intelligence quotient (IQ) of $<70 .^{2}$ A similar picture is emerging from the growing cohort of patients surviving into adulthood. ${ }^{11}$ Patients with hypoplastic left heart syndrome appear to be significantly more affected than patients with other univentricular conditions in terms of both neurocognitive ${ }^{3}$ and educational and behavioural difficulties.

Despite recent advances in surgical and bypass techniques used in the treatment of hypoplastic left heart syndrome such as off-bypass neonatal palliation, anterograde regional cerebral perfusion during cardiac arrest, and near-infrared cerebral saturation monitoring, ${ }^{9,23}$ neurodevelopmental outcomes following the Norwood procedure have remained relatively static. ${ }^{24,25}$ As early as the 1990s, however, evidence began to emerge of clinical neurological and electroencephalographic impairments in newborn babies with CHD even before intervention, ${ }^{26,27}$ which were strongly linked to later neuromotor and cognitive impairments, functional limitations, and impaired quality of life. ${ }^{1,27}$ There is now a growing body of evidence that the neurodevelopmental outcomes for hypoplastic left heart syndrome patients are not solely determined by clinical, surgical, and interventional factors after birth; a significant burden of pre-existing brain abnormalities exists even before any attempts at surgical palliation.

\section{Effects of hypoplastic left heart syndrome on fetal circulation}

In the normal fetus, both the right and the left sides of the heart support the systemic circulation, atrial and arterial shunts are present, and the placental circulation provides both oxygenation and nutritional support (Fig 1a). ${ }^{18}$ Placental blood is preferentially streamed via the foramen ovale to the left heart, which supplies the superior portion of the systemic circulation, delivering oxygen-rich and nutritionally rich blood to the ascending aorta, carotid arteries, and the developing brain.

The effects of abnormal cardiovascular anatomy on the fetal circulation have been considered in detail since the $1970 \mathrm{~s} .{ }^{28}$ Depending on the nature of the anatomical disturbance, flow rates in the ascending aorta can be increased or decreased, and the preferential shunting of oxygenated blood to the head and neck vessels may be disrupted.

In the case of hypoplastic left heart syndrome, flow through the often profoundly underdeveloped left ventricle is either reduced or absent. The ascending 
(a)

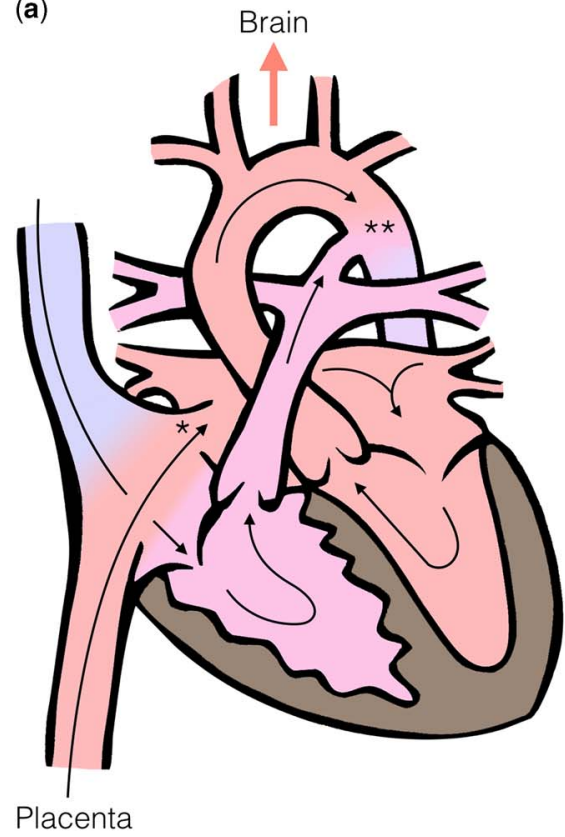

(b) Brain

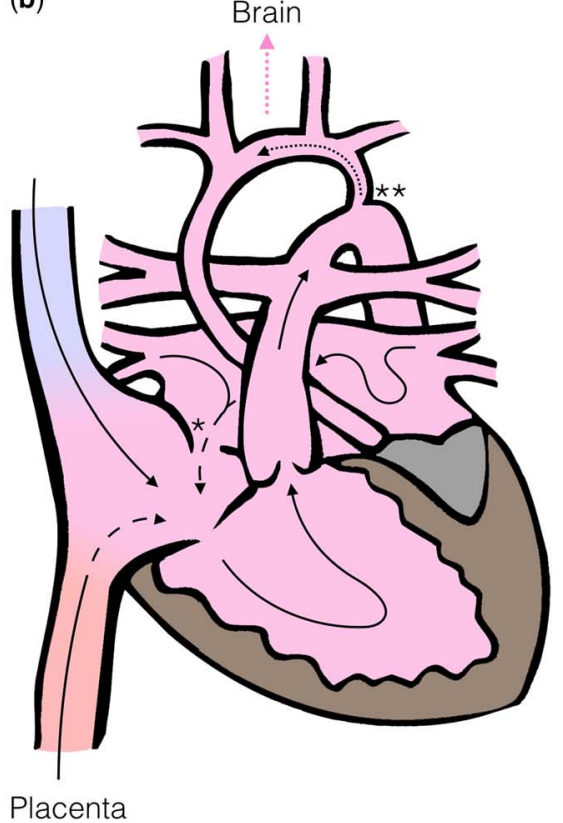

Figure 1.

In the normal fetal circulation (a), oxygenated blood is shunted to the left heart via the foramen ovale (*), allowing nutrient and oxygen rich blood from the placenta to be preferentially directed to the cerebral circulation. In the fetus with HLHS (b) the left sided structures are underdeveloped, leading to reversed flow at the foramen ovale (*) and aortic isthmus (**), which may be severely hypoplastic. This leads to reduced oxygen and substrate delivery to the developing brain.

aorta and the aortic arch are also frequently hypoplastic and dependent on retrograde filling via the arterial duct (Fig 1b). In addition, the obligatory intracardiac mixing of oxygenated and deoxygenated blood and loss of venous streaming results in blood reaching the brain having a lower oxygen and nutritional content than in the normal fetal circulation. ${ }^{29}$

\section{Effects of hypoplastic left heart syndrome on fetal brain development}

Normal fetal brain growth and development is a function of adequate oxygen and substrate delivery, and the fetus has complex mechanisms for autoregulation of cerebrovascular resistance to increase oxygen delivery and meet cerebral energy requirements. ${ }^{30}$

Intrauterine growth restriction ${ }^{31}$ and particularly microcephaly ${ }^{32}$ are associated with hypoplastic left heart syndrome, with the latter potentially reflecting abnormal brain development in utero. In hypoplastic left heart syndrome, the entire cerebral circulation is dependent on the retrograde flow from the arterial duct through the aortic isthmus, with potential restriction to blood flow to the aortic arch and head and neck vessels (Fig 1b). ${ }^{33}$ Microcephaly is known to be associated with reduced cerebral blood flow before birth, ${ }^{34,35}$ and has also been independently associated with a diminutive ascending aorta in hypoplastic left heart syndrome patients. ${ }^{7}$ Cerebral autoregulatory mechanisms offer further insight: during periods of hypoxic stress, the normal fetus compensates by reducing cerebrovascular resistance to re-distribute blood flow to the upper body and brain. This mechanism can be identified prenatally by Doppler ultrasound interrogation showing increased diastolic velocities in the cerebral arteries and reduced diastolic velocities in the descending aorta and umbilical artery. ${ }^{36}$ Normative indices of resistance and pulsatility have been established, and $z$-scores are available. ${ }^{30}$ Several studies have shown lower than normal cerebral artery/umbilical artery resistance ratios in patients with hypoplastic left heart syndrome, ${ }^{37,38}$ suggesting activation of brain-sparing mechanisms in an attempt to increase cerebral blood flow. Indeed, the presence of reversed flow across the aortic isthmus may in itself be an important independent factor in triggering this response. ${ }^{39}$ Maintaining the ability to re-distribute blood flow in this manner may be important; in one retrospectively analysed study of the echocardiograms of 134 hypoplastic left heart syndrome and other single-ventricle fetuses, lower fetal cerebrovascular resistance was positively associated with better neurodevelopmental scores in later life. ${ }^{40}$

In addition to microcephaly, both structural brain abnormalities and white matter injury have been identified in patients with hypoplastic left heart syndrome. Neuropathological studies of the brains of fetuses with hypoplastic left heart syndrome have 
shown structural abnormalities such as agenesis of the corpus callosum and holoprosencephaly, ${ }^{41}$ as well as significant white matter injuries. ${ }^{32}$ Prenatal and postnatal MRI studies have further defined structural abnormalities, demonstrating incomplete closure of the operculum, ventriculomegaly, and cerebral atrophy. Multiple patterns of brain injury have also been described, including haemorrhagic and thromboembolic infarction, cerebral venous thromboses, periventricular leukomalacia, and grey matter injury. ${ }^{1,16,42,43,44}$

Although fetal brain injuries detected by MRI have been shown to be strong predictors of neurodevelopmental disability in other high-risk term and preterm newborns, ${ }^{45}$ precisely how preoperative brain injury correlates to later impairment in hypoplastic left heart syndrome patients is not yet clear. In the largest study to date, no direct correlation was found between preoperative brain injury across a range of $\mathrm{CHD}$ diagnoses and neurodevelopmental outcomes at 12 months, although over a third of patients either did not survive or failed to return for testing. ${ }^{46}$ Further longitudinal studies are needed, both to provide a uniform means of sub-classifying preoperative brain injuries and to correlate these with later performance across all neurodevelopmental domains.

Advanced fetal MRI techniques have offered further insights into abnormal brain development in hypoplastic left heart syndrome. MRI spin-label perfusion studies have shown significantly reduced cerebral blood flow in the months before surgery. ${ }^{47}$ Magnetic resonance spectroscopy enables in vivo quantification of certain chemical compounds and metabolites. Using this technique, term newborns with single-ventricle hearts were shown to have increased cerebral lactate levels, which may indicate inadequate cerebral metabolism before surgery. ${ }^{16}$ In addition, by measuring the levels of $N$-acetylaspartate, a marker of neuronal integrity, lactate/ choline ratios, and average diffusivity measured by diffusion tensor imaging, patients with $\mathrm{CHD}$ have been found to have features of delayed brain maturation versus controls of around 1 month, re-asserting that inefficient cerebral substrate delivery is likely to begin before birth. ${ }^{30}$ Indeed, by using similar techniques on the fetal brain, prenatal MRI studies have identified that brain metabolism, growth, and maturation are particularly delayed in the third trimester, a period of increased synapse formation, myelination, and high metabolic demand. A reduction in combined ventricular output across the aortic valve was independently associated with a reduction in total brain volume, and absence of anterograde arch flow was associated with delayed brain maturity. ${ }^{48}$ In contrast to overt brain injury, markers of brain immaturity appear to correlate more convincingly to later neurodevelopmental status, with severity at birth shown to be a good predictor of neurodevelopmental impairment at 2 years of age after cardiac surgery. ${ }^{49}$ Furthermore, studies in adolescents have also shown a direct correlation between brain volume and neurodevelopmental outcomes. ${ }^{50}$

Finally, although it has been demonstrated that patients with delayed brain maturation are at particular risk of sustaining new white matter injuries postoperatively, ${ }^{51}$ pre-existing brain injury does not appear to worsen following surgery, adding more weight to the argument that surgical factors may play a lesser role than that previously suspected. ${ }^{43,52}$

\section{Opportunities for the future}

Alongside exciting developments in three and fourdimensional fetal ultrasound such as spatio-temporal image correlation imaging, ${ }^{53}$ fetal MRI is quickly gaining ground as an important complementary imaging tool, with the additional resources required increasingly offset by a growing number of fetal indications $^{54}$ and rapidly developing MRI infrastructure. $^{55}$ By combining established techniques with advanced motion-correction algorithms, ${ }^{56}$ retrospectively gated flow measurements, ${ }^{59}$ and non-invasive estimates of intravascular oxygen saturations, ${ }^{58}$ there is the potential to generate a detailed anatomical and physiological profile of the fetal circulation in CHD.

In addition, centres performing prenatal brain imaging are enhancing our understanding of both gross brain injury and subtle patterns of disordered brain development and oxygen uptake and metabolism, which may accompany these disruptions. ${ }^{58-60}$ In correlating these data to later neurodevelopmental impairments, it may be possible to establish hypoplastic left heart syndrome cardiac phenotypes, patterns of disordered brain development, and categories of brain injury that confer a higher risk for later neurodevelopmental problems. Addressing modifiable risk factors via tailored neuroprotective strategies, ${ }^{9}$ novel monitoring techniques, ${ }^{23}$ and judicious attention to anaesthetic load, ${ }^{46}$ in conjunction with subsequent early access to developmental support programmes, could help improve longer-term outcomes. $^{20}$

Further into the future, as we begin to understand the complex fetoplacental interactions in fetuses with $\mathrm{CHD}$, manipulation of the fetal circulation via experimental interventions such as maternal hyperoxygenation may provide a non-invasive means of improving cerebral oxygen and substrate delivery in selected patients. ${ }^{61}$ Finally, the use of direct fetal cardiac intervention, currently being explored by a 
small number of centres for a limited set of indications, ${ }^{62}$ may develop a role as our understanding and expertise develop in this area.

\section{Impact on fetal counselling}

Counselling the prospective parents of a fetus with hypoplastic left heart syndrome poses many challenges for physicians, counsellors, and the parents themselves. Potential management options may include a variety of approaches, including variants of the Norwood procedure, the hybrid procedure, compassionate care after birth, primary transplantation, or termination of the pregnancy. As such, an antenatal diagnosis of hypoplastic left heart syndrome demands tailored, sensitive, and intuitive counselling, relevant to each parent's level of understanding and cultural, moral, and emotional needs. ${ }^{63}$ Determining fetal phenotypes at high risk not only of surgical mortality but also of poor neurodevelopmental outcome may allow for more comprehensive counselling for carers, in whom predictions based on mortality risk alone have been shown to have little effect on antenatal decision making. ${ }^{19}$ A more comprehensive risk profile that includes neurodevelopmental risk factors may be far more relevant for prospective parents, in terms of both antenatal decision making and longer-term expectations.

\section{Summary}

Neonates with hypoplastic left heart syndrome have a unique set of neurological vulnerabilities before they even encounter the operating theatre, with the potential for impaired cerebral blood flow, oxygen and nutrient delivery, leading to delayed or disordered brain development, and pre-existing brain injuries. These factors are present before any intervention, and are almost certainly a substantial contributor to the many factors that may contribute to adverse neurodevelopmental outcomes in later childhood and adulthood.

The use of novel cardiac neuroimaging before and after birth is enhancing our ability to define specific hypoplastic left heart syndrome phenotypes at risk of developing significant neurodevelopmental impairments, potentially providing opportunities to tailor neuroprotective strategies, enable early access to structured neurodevelopmental support programmes, and enhance fetal counselling.

\section{Acknowledgement}

Open access for this article was funded by King's College London.

\section{Financial Support}

This research received no specific grant from any funding agency or from commercial or not-for-profit sectors.

\section{Conflicts of Interest}

None.

\section{References}

1. Donofrio MT, Duplessis AJ, Limperopoulos C. Impact of congenital heart disease on fetal brain development and injury. Curr Opin Pediatr 2011; 23: 502-511.

2. Mahle WT, Wernovsky G. Neurodevelopmental outcomes in hypoplastic left heart syndrome. Semin Thorac Cardiovasc Surg Pediatr Card Surg Annu 2004; 7: 39-47.

3. Sarajuuri A, Jokinen E, Puosi R, et al. Neurodevelopment in children with hypoplastic left heart syndrome. J Pediatr 2010; 157: 414-420; e414.

4. Gaynor JW, Gerdes M, Nord AS, et al. Is cardiac diagnosis a predictor of neurodevelopmental outcome after cardiac surgery in infancy? Cardiol Young 2010; 140: 1230-1237.

5. Davidson J, Gringras P, Fairhurst C, Simpson J. Physical and neurodevelopmental outcomes in children with single-ventricle circulation. Arch Dis Child 2015; 100: 449-453.

6. Gaynor JW, Stopp C, Wypij D, et al. Neurodevelopmental outcomes after cardiac surgery in infancy. Pediatrics 2015; 135: 816-825.

7. Shillingford AJ, Ittenbach RF, Marino BS, et al. Aortic morphometry and microcephaly in hypoplastic left heart syndrome. Cardiol Young 2007; 17: 189-195.

8. Wypij D, Newburger JW, Rappaport LA. The effect of duration of deep hypothermic circulatory arrest in infant heart surgery on late neurodevelopment: the Boston Circulatory Arrest Trial. J Thorac Cardiovasc Surg 2003; 126: 1397-1403.

9. Hirsch JC, Jacobs ML, Andropoulos D, et al. Protecting the infant brain during cardiac surgery: a systematic review. Ann Thorac Surg 2012; 94: 1365-1373.

10. von Rhein M, Dimitropoulos A, Valsangiacomo Buechel ER, Landolt MA, Latal B. Risk factors for neurodevelopmental impairments in school-age children after cardiac surgery with fullflow cardiopulmonary bypass. J Thorac Cardiovasc Surg 2012; 144 : 577-583.

11. Kempny A, Dimopoulos K, Gatzoulis MA. Single-ventricle physiology in the UK: an ongoing challenge of growing numbers and of growing complexity of congenital heart disease. Heart 2014; 100: $1315-1316$.

12. Ballweg JA, Wernovsky G, Gaynor JW. Neurodevelopmental outcomes following congenital heart surgery. Pediatr Cardiol 2007; 28: 126-133.

13. Laskowitz DT, Lynch JR, Warner DS. Apolipoprotein E modulates the CNS response to injury. J Neurochem 2002; 81 (Suppl 1): 31.

14. Forbess JM, Visconti KJ, Bellinger DC, Howe RJ. Neurodevelopmental outcomes after biventricular repair of congenital heart defects. J Thorac Cardiovasc Surg 2002; 123: 631-639.

15. Kucik JE, Nembhard WN, Donohue P, et al. Community socioeconomic disadvantage and the survival of infants with congenital heart defects. Am J Public Health 2014; 104: e150-e157.

16. Miller SP, McQuillen PS, Hamrick S, et al. Abnormal brain development in newborns with congenital heart disease. $\mathrm{N}$ Engl $\mathrm{J}$ Med 2007; 357: 1928-1938.

17. Owen M, Shevell M, Majnemer A, Limperopoulos C. Abnormal brain structure and function in newborns with complex congenital heart defects before open heart surgery: a review of the evidence. J Child Neurol 2011; 26: 743-755. 
18. Hunter LE, Simpson JM. Prenatal screening for structural congenital heart disease. Nat Rev Cardiol 2014; 11: 323-334.

19. Rychik J, Szwast A, Natarajan S, et al. Perinatal and early surgical outcome for the fetus with hypoplastic left heart syndrome: a 5-year single institutional experience. Ultrasound Obstet Gynecol 2010; 36: 465-470.

20. Marino BS. New concepts in predicting, evaluating, and managing neurodevelopmental outcomes in children with congenital heart disease. Curr Opin Pediatr 2013; 25: 574-584.

21. Norwood WI, Lang P, Casteneda AR, Campbell DN. Experience with operations for hypoplastic left heart syndrome. J Thorac Cardiovasc Surg 1981; 82: 511-519.

22. Majnemer A, Limperopoulos C, Shevell MI, Rohlicek C, Rosenblatt B, Tchervenkov C. A new look at outcomes of infants with congenital heart disease. Pediatr Neurol 2009; 40: 197-204.

23. Hoffman GM, Brosig CL, Mussatto KA, Tweddell JS, Ghanayem NS. Perioperative cerebral oxygen saturation in neonates with hypoplastic left heart syndrome and childhood neurodevelopmental outcome. J Thorac Cardiovasc Surg 2013; 146: 1153-1164.

24. Knirsch W, Liamlahi R, Hug MI, et al. Mortality and neurodevelopmental outcome at 1 year of age comparing hybrid and Norwood procedures. Eur J Cardiothorac Surg 2012; 42: 33-39.

25. Newburger JW, Sleeper LA, Bellinger DC, et al. Early developmental outcome in children with hypoplastic left heart syndrome and related anomalies: the single ventricle reconstruction trial. Circulation 2012; 125: 2081-2091.

26. Limperopoulos C, Majnemer A, Shevell MI, Rosenblatt B, Rohlicek C, Tchervenkov C. Neurologic status of newborns with congenital heart defects before open heart surgery. Pediatrics 1999; 103: 402-408.

27. Limperopoulos C, Majnemer A, Shevell MI, Rosenblatt B, Rohlicek C, Tchervenkov C. Neurodevelopmental status of newborns and infants with congenital heart defects before and after open heart surgery. J Pediatr 2000; 137: 638-645.

28. Heymann MA, Rudolph AM. Effects of congenital heart disease on fetal and neonatal circulations. Prog Cardiovasc Dis 1972; 15: $115-143$.

29. Rosenthal GL. Patterns of prenatal growth among infants with cardiovascular malformations: possible fetal hemodynamic effects. Am J Epidemiol 1996; 143: 505-513.

30. Donofrio MT, Massaro AN. Impact of congenital heart disease on brain development and neurodevelopmental outcome. Int J Pediatr 2010; 2010: 359390.

31. Petrossian RA, Kuehl KS, Loffredo CA. Relationship of birth weight with congenital cardiovascular malformations in a population-based study. Cardiol Young 2015; 25: 1086-1092.

32. Hinton RB, Andelfinger G, Sekar P, et al. Prenatal head growth and white matter injury in hypoplastic left heart syndrome. Pediatr Res 2008; 64: 364-369.

33. Sakazaki S, Masutani S, Sugimoto M, et al. Oxygen supply to the fetal cerebral circulation in hypoplastic left heart syndrome: a simulation study based on the theoretical models of fetal circulation. Pediatr Cardiol 2014; 36: 677-684.

34. Donofrio MT, Bremer YA, Schieken RM, et al. Autoregulation of cerebral blood flow in fetuses with congenital heart disease: the brain sparing effect. Pediatr Cardiol 2003; 24: 436-443.

35. Arduini M, Rosati P, Caforio L, et al. Cerebral blood flow autoregulation and congenital heart disease: possible causes of abnormal prenatal neurologic development. J Matern Fetal Neonatal Med 2011; 24: 1208-1211.

36. Dubiel M, Gunnarsson GO, Gudmundsson S. Blood redistribution in the fetal brain during chronic hypoxia. Ultrasound Obstet Gynecol 2002; 20: 117-121.

37. Szwast A, Tian Z, McCann M, Soffer D, Rychik J. Comparative analysis of cerebrovascular resistance in fetuses with single-ventricle congenital heart disease. Ultrasound Obstet Gynecol 2012; 40: $62-67$.
38. Masoller N, Sanz-Cortés M, Crispi F, et al. Mid-gestation brain Doppler and head biometry in fetuses with congenital heart disease predict abnormal brain development at birth. Ultrasound Obstet Gynecol 2016; 47: 65-73.

39. Yamamoto Y, Khoo NS, Brooks PA, Savard W, Hirose A, Hornberger LK. Severe left heart obstruction with retrograde arch flow influences fetal cerebral and placental blood flow. Ultrasound Obstet Gynecol 2013; 42: 294-299.

40. Williams IA, Fifer C, Jaeggi E, Levine JC, Michelfelder EC, Szwast AL. The association of fetal cerebrovascular resistance with early neurodevelopment in single ventricle congenital heart disease. Am Heart J 2013; 165: 544-550; e541.

41. Glauser TA, Rorke LB, Weinberg PM, Clancy RR. Congenital brain anomalies associated with the hypoplastic left heart syndrome. Pediatrics 1990; 85: 984-990.

42. Licht DJ, Shera DM, Clancy RR, et al. Brain maturation is delayed in infants with complex congenital heart defects. J Thorac Cardiovasc Surg 2009; 137: 529-536; discussion 536-537.

43. Block AJ, McQuillen PS, Chau V, et al. Clinically silent preoperative brain injuries do not worsen with surgery in neonates with congenital heart disease. J Thorac Cardiovasc Surg 2010; 140: 550-557.

44. Peyvandi S, De Santiago V, Chakkarapani E, et al. Association of prenatal diagnosis of critical congenital heart disease with postnatal brain development and the risk of brain injury. JAMA Pediatr 2016; 170: e154450.

45. Massaro AN. MRI for neurodevelopmental prognostication in the high-risk term infant. Semin Perinatol 2015; 39: 159-167.

46. Andropoulos DB, Ahmad HB, Haq T, et al. The association between brain injury, perioperative anesthetic exposure, and 12-month neurodevelopmental outcomes after neonatal cardiac surgery: a retrospective cohort study. Paediatr Anaesth 2014; 24: 266-274.

47. Licht DJ, Wang J, Silvestre DW, et al. Preoperative cerebral blood flow is diminished in neonates with severe congenital heart defects. J Thorac Cardiovasc Surg 2004; 128: 841-849.

48. Limperopoulos C, Tworetzky W, McElhinney DB, et al. Brain volume and metabolism in fetuses with congenital heart disease: evaluation with quantitative magnetic resonance imaging and spectroscopy. Circulation 2010; 121: 26-33.

49. Beca J, Gunn JK, Coleman L, et al. New white matter brain injury after infant heart surgery is associated with diagnostic group and the use of circulatory arrest. Circulation 2013; 127: 971-979.

50. von Rhein M, Buchmann A, Hagmann C, et al. Brain volumes predict neurodevelopment in adolescents after surgery for congenital heart disease. Brain 2014; 137 (Pt 1): 268-276.

51. Andropoulos DB, Hunter JV, Nelson DP, et al. Brain immaturity is associated with brain injury before and after neonatal cardiac surgery with high-flow bypass and cerebral oxygenation monitoring. J Thorac Cardiovasc Surg 2010; 139: 543-556.

52. Wernovsky G. Current insights regarding neurological and developmental abnormalities in children and young adults with complex congenital cardiac disease. Cardiol Young 2006; 16 (Suppl 1): 92-104.

53. Ahmed BI. The new $3 \mathrm{D} / 4 \mathrm{D}$ based spatio-temporal imaging correlation (STIC) in fetal echocardiography: a promising tool for the future. J Matern Fetal Neonatal Med 2014; 27: 1163-1168.

54. Gholipour A, Estroff JA, Barnewolt CE, et al. Fetal MRI: a technical update with educational aspirations. Concepts Magn Reson Part A Bridg Educ Res 2014; 43: 237-266.

55. Svenson M, Steele P. NHS imaging and radiodiagnostic activity, 2013-2014. Retrieved March 12, 2016, from england.nhs.uk/ statistics/statistical-work-areas/diagnostics-waiting-times-andactivity/imaging-and-radiodiagnostics-annual-data/

56. Kainz B, Malamateniou C, Murgasova M, et al. Motion corrected 3D reconstruction of the fetal thorax from prenatal MRI. Med Image Comput Comput Assist Interv 2014; 17 (Pt 2): 284-291.

57. Seed M, van Amerom JFP, Yoo SJ, et al. Feasibility of quantification of the distribution of blood flow in the normal human fetal 
Vol. 27, No. 2 Lloyd et al: The neurodevelopmental implications of hypoplastic left heart syndrome in the fetus

circulation using CMR: a cross-sectional study. J Cardiovasc Magn Reson 2012; 14: 79.

58. Sun L, Macgowan CK, Sled JG, et al. Reduced fetal cerebral oxygen consumption is associated with smaller brain size in fetuses with congenital heart disease. Circulation 2015; 131: $1313-1323$.

59. Rutherford M, Ramenghi LA, Edwards AD, et al. Assessment of brain tissue injury after moderate hypothermia in neonates with hypoxic-ischaemic encephalopathy: a nested substudy of a randomised controlled trial. Lancet Neurol 2010; 9: 39-45.
60. Rutherford M, Biarge MM, Allsop J, Counsell S. MRI of perinatal brain injury. Pediatr Radiol 2010; 40: 819-833.

61. Porayette P, Sun L, Jaeggi E, et al. MRI reveals hemodynamic changes with acute maternal hyperoxygenation in human fetuses with and without congenital heart disease. J Cardiovasc Magn Reson 2015; 17 (Suppl 1): O55.

62. McElhinney DB, Tworetzky W, Lock JE. Current status of fetal cardiac intervention. Circulation 2010; 121: 1256-1263.

63. Allan LD, Huggon IC. Counselling following a diagnosis of congenital heart disease. Prenat Diagn 2004; 24: 1136-1142. 